\title{
Fabry disease screening in high-risk populations in Japan: a nationwide study
}

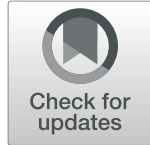

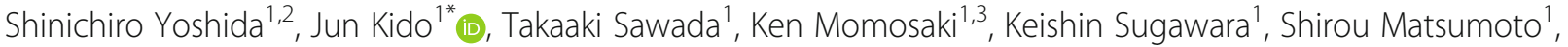
Fumio Endo ${ }^{1,3}$ and Kimitoshi Nakamura ${ }^{1}$

\begin{abstract}
Background: Fabry disease (FD) is a X-linked inherited disorder caused by mutations in the GLA gene, which results in the deficiency of a-galactosidase A ( $\mathrm{a}-\mathrm{Gal}$ A). This leads to the progressive accumulation of metabolites, which can cause multisystemic dysfunction. A recent screening study among neonates reported an increase in the incidence of FD, and numerous FD patients remain undiagnosed or even misdiagnosed. Therefore, this study aimed to identify patients with FD by performing high-risk screening in 18,135 individuals, enrolled from October 2006 to March 2019, with renal, cardiac, or neurological manifestations from all prefectures in Japan. A total of 601 hospitals participated in this study.

Results: Low a-Gal A activity was detected in 846 individuals, with 224 of them diagnosed with FD by GLA sequencing. Cases with a family history of FD $(n=64)$ were also subjected to sequencing, without a-Gal A assay, as per individual request, and 12 of them were diagnosed with a variant of FD. A total of 236 patients with FD (97 males and 139 females) were identified from among 18,199 participants. A total of 101 GLA variants, including 26 novel variants, were detected in the 236 patients with FD from 143 families, with 39 amenable variants (39\%) and 79 of the 236 patients (33\%) suitable for migalastat treatment.
\end{abstract}

Conclusions: From among 18,199 participants, 101 GLA variants, including 26 novel variants, were identified in the 236 patients with FD from 143 families. Migalastat was identified as a suitable treatment option in $33 \%$ of the patients with FD and 39\% of the GLA variants were detected as amenable. Therefore, the simple screening protocol using dried blood spots that was performed in this study could be useful for early diagnosis and selection of appropriate treatments for FD in high-risk and underdiagnosed patients with various renal, cardiac, or neurological manifestations.

Keywords: Fabry disease, High-risk screening, a-Galactosidase a, GLA gene, Novel variants

\section{Background}

Fabry disease (FD; OMIM 301500) is an inherited Xlinked disorder caused by mutations in the GLA gene, which encodes the lysosomal enzyme $\alpha$-galactosidase A ( $\alpha$-Gal A; EC 3.2.1.22). To date, 516 and 612 GLA variants have been incorporated into the Fabry-database (Fabrydatabase.org, ver. 3.2.2, last updated on February 15, 2019)

\footnotetext{
* Correspondence: kidojun@kuh.kumamoto-u.ac.jp

'Department of Pediatrics, Graduate School of Medical Sciences, Kumamoto University, 1-1-1 Honjo, Chuo-ku, Kumamoto City, Kumamoto 860-8556, Japan

Full list of author information is available at the end of the article
}

[1] and ClinVar (http://www.ncbi.nlm.nih.gov/clinvar) [2], respectively. The functional deficiency of $\alpha-G a l$ A results in the progressive accumulation of metabolites, such as globotriaosylceramide in lysosomes, biological fluids, and the vascular endothelium, which can cause disease manifestations in the skin, eyes, kidneys, ears, lungs, heart, and brain [3-5]. FD patients who have very low $\alpha$-Gal A activity exhibit the classic phenotype and are generally asymptomatic in early childhood [6, 7]. In contrast, FD patients with residual $\alpha$-Gal A activity exhibit milder clinical manifestations and onset occurs later than in those with the classic phenotype. Heterozygous women with pathogenic

(c) The Author(s). 2020 Open Access This article is licensed under a Creative Commons Attribution 4.0 International License, which permits use, sharing, adaptation, distribution and reproduction in any medium or format, as long as you give appropriate credit to the original author(s) and the source, provide a link to the Creative Commons licence, and indicate if changes were made. The images or other third party material in this article are included in the article's Creative Commons licence, unless indicated otherwise in a credit line to the material. If material is not included in the article's Creative Commons licence and your intended use is not permitted by statutory regulation or exceeds the permitted use, you will need to obtain permission directly from the copyright holder. To view a copy of this licence, visit http://creativecommons.org/licenses/by/4.0/ The Creative Commons Public Domain Dedication waiver (http://creativecommons.org/publicdomain/zero/1.0/) applies to the data made available in this article, unless otherwise stated in a credit line to the data. 
GLA variants are not only carriers but also express wide manifestation spectra, ranging from asymptomatic to as severe as those of the classic phenotype, depending on random X-chromosomal inactivation [8].

Clinical manifestations are multisystemic, including limb pain, acroparesthesia, angiokeratoma, anhidrosis, and corneal opacity in childhood, with a progression to major organ involvement in adulthood, such as proteinuria, impaired renal function, cardiomyopathy, and stroke. Because these manifestations are frequently observed in individuals with diabetes, hypertension, and arteriosclerosis, which are nonspecific, this might lead to a delayed or an incorrect diagnosis $[9,10]$. Recent newborn screening (NBS) studies, including our previous study [11], reported that the incidence of FD was as high as 1:1600 to 1:8485 in live births $[12,13]$. Therefore, the prevalence of FD is underestimated, with evidence suggesting that there are many undiagnosed or misdiagnosed FD patients.

Enzyme replacement therapy (ERT) is now available in Japan and three products are on the market, namely Fabrazyme $^{\oplus}$ (Sanofi Genzyme), Replagal ${ }^{\circ}$ (Shire), Agalsidase beta BS (JCR). Moreover, an oral pharmacological chaperone, migalastat (Galafold ${ }^{\circledR}$; Amicus Therapeutics), has become available for specific pathogenic GLA variants, i.e., migalastat-amenable GLA variants [14]. ERT can decelerate renal deterioration and the progression of cardiomyopathy, thereby delaying morbidity and mortality [15]. Migalastat has the same effects on renal function as ERT [16]. Early treatment is essential to preserve organ function and prevent progression of the disease. The high-risk screening for FD is considered a practical strategy for early treatment.

Nakagawa et al. [17] reported partial results regarding high-risk Japanese patients in the Hokkaido prefecture with cardiac, renal, or neurological manifestations. This study aimed to identify undiagnosed patients with FD by performing high-risk screening among 18,199 individuals with renal, cardiac, or neurological manifestations, from all of the prefectures in Japan, and to assess the effectiveness of our simple screening protocol for the definite diagnosis of FD in the aforementioned high-risk groups.

\section{Results}

\section{High-risk screening for Fabry disease}

The demographic characteristics of the enrolled individuals are summarized in Table 1 . Of the 18,135

Table 1 Demographic characteristics of enrolled individuals

\begin{tabular}{|c|c|c|c|c|c|c|c|}
\hline \multirow[t]{3}{*}{ Group } & \multicolumn{4}{|l|}{ Enrolled } & \multicolumn{3}{|c|}{ Variant detected } \\
\hline & \multirow[t]{2}{*}{ Gender } & \multirow[t]{2}{*}{$n$} & \multicolumn{2}{|c|}{ Age (year) } & \multirow[t]{2}{*}{ n (\%)\$ } & \multicolumn{2}{|c|}{ Age (year) } \\
\hline & & & Median & IQR & & Median & IQR \\
\hline \multirow[t]{3}{*}{ a } & M & 5505 & 64 & $55-73$ & $19(0.45)$ & 49 & $40-55$ \\
\hline & $\mathrm{F}$ & 3090 & 66 & $56-74$ & $9(0.38)$ & 58 & NC \\
\hline & M or F & 228 & & & & & \\
\hline \multirow[t]{3}{*}{ b } & M & 2853 & 61 & $48-71$ & $17(0.77)$ & 47 & $37-56$ \\
\hline & $\mathrm{F}$ & 1176 & 67 & $54-75$ & $12(1.33)$ & 47.5 & $33.75-53.75$ \\
\hline & $M$ or $F$ & 28 & & & & & \\
\hline \multirow[t]{3}{*}{ c } & M & 1926 & 67 & $54-77$ & $3(0.20)$ & $\mathrm{NC}$ & NC \\
\hline & $\mathrm{F}$ & 968 & 72 & $53-82$ & $2(0.27)$ & NC & NC \\
\hline & Mor F & 181 & & & & & \\
\hline \multirow[t]{3}{*}{$d$} & M & 522 & 13 & $8-31$ & $20(4.98)$ & 13 & $11-18.5$ \\
\hline & $\mathrm{F}$ & 371 & 14 & $9-41$ & $10(3.50)$ & 13 & $9-25$ \\
\hline & Mor F & 1 & & & & & \\
\hline \multirow[t]{3}{*}{ e } & M & 333 & 28 & $11-45$ & 32 (12.49) & 20 & 13-39 \\
\hline & $\mathrm{F}$ & 378 & 36 & $21-49$ & 96 (33.02) & 38 & $18.75-49.75$ \\
\hline & $\mathrm{M}$ or $\mathrm{F}$ & 4 & & & & & \\
\hline \multirow[t]{4}{*}{$f$} & M & 350 & 46 & $16-63$ & $3(1.11)$ & NC & NC \\
\hline & $\mathrm{F}$ & 206 & 43 & $14-64$ & $1(0.63)$ & NC & NC \\
\hline & M or F & 15 & & & & & \\
\hline & Total & 18,135 & & & 224 & & \\
\hline
\end{tabular}


individuals that were screened, 8823 had renal manifestations (5505 males, 3090 females, and 228 did not provide information regarding sex), 4057 presented signs of cardiac manifestations (2853 males, 1176 females; 28 did not provide information regarding sex), 3075 evidenced central neurological manifestations (1926 males, 968 females; 181 did not provide information regarding sex), 894 experienced peripheral neurological manifestations (522 males, 371 females; 1 did not provide information regarding sex), 715 had a family history of FD (333 males, 378 females; 4 did not provide information regarding sex), and 571 were classified as "other" (350 males, 206 females; 15 did not provide information regarding sex). The flow chart and results of the high-risk screening program for FD are presented in Fig. 1. The 2553 individuals, who had low $\alpha$-Gal A activity, were recalled and 1965 of them proceeded to the second $\alpha$ Gal A assay. Figures 2 and S1 shows the distribution of $\alpha$-Gal A activity in the first assay in Method I. Approximately 4 and $28 \%$ of male and female patients, respectively, fell below the cutoff value. The median $\alpha$-Gal A activity obtained using Method I was 24.47, 24.50, and 24.06 (AgalU) in among all individuals, men, and women, respectively (Fig. 2a, b, and c). The median $\alpha-$ Gal A activity obtained using Method II was 57.50, 62.54, and 45.53 (AgalU) among all individuals, men, and women, respectively. From Methods I and II, 846 individuals were identified and 224 of them were diagnosed with FD by GLA sequencing. No GLA gene variants were identified in 650 individuals, whereas 52 individuals presented with a possible functional polymorphism allele, p.E66Q. Of the 224 individuals that presented with GLA gene variants, 28 (19 male, 9 female), 29 (17 male, 12 female), 5 (3 male, 2 female), 30 (20 male, 10 female), 128 (32 male, 96 female), and 4 (3 male, 1 female) variants were identified in the renal, cardiac, central neurological, peripheral neurological, family history, and "other" groups, respectively (Table 1). The prevalence of FD was $0.42 \%$ (male: $0.45 \%$, female: $0.38 \%$ ), $0.94 \%$ (male: $0.77 \%$, female: $1.33 \%$ ), $0.22 \%$ (male: $0.20 \%$, female: $0.27 \%$ ), $4.37 \%$ (male: $4.98 \%$, female: $3.50 \%$ ), and $23.40 \%$ (male: $12.49 \%$, female: $33.02 \%$ ) in the renal, cardiac, central neurological, peripheral neurological, and family history groups, respectively. The median age was 49 years for men and 58 years for women, 47 years for men and 47.5 years for women, 13 years for both men and women, and 20 years for men and 38 years for women in the renal, cardiac, peripheral neurological, and family history groups, respectively. Individuals with a family history of FD $(n=64)$ were also subjected to sequencing analysis, without $\alpha-\mathrm{Gal} A$ assay, as per individual request. GLA gene variants were detected in 12 ( 3 males and 9 females) of the 64 individuals. Therefore, a total of 236 FD patients were detected from 18,199 individuals in this high-risk screening study.

\section{GLA variants detected in the FD patients}

Herein, 101 GLA variants were detected in 236 patients from 143 families (Table 2). Of these, 39 (39\%) were amenable and 79 of the 236 patients were considered

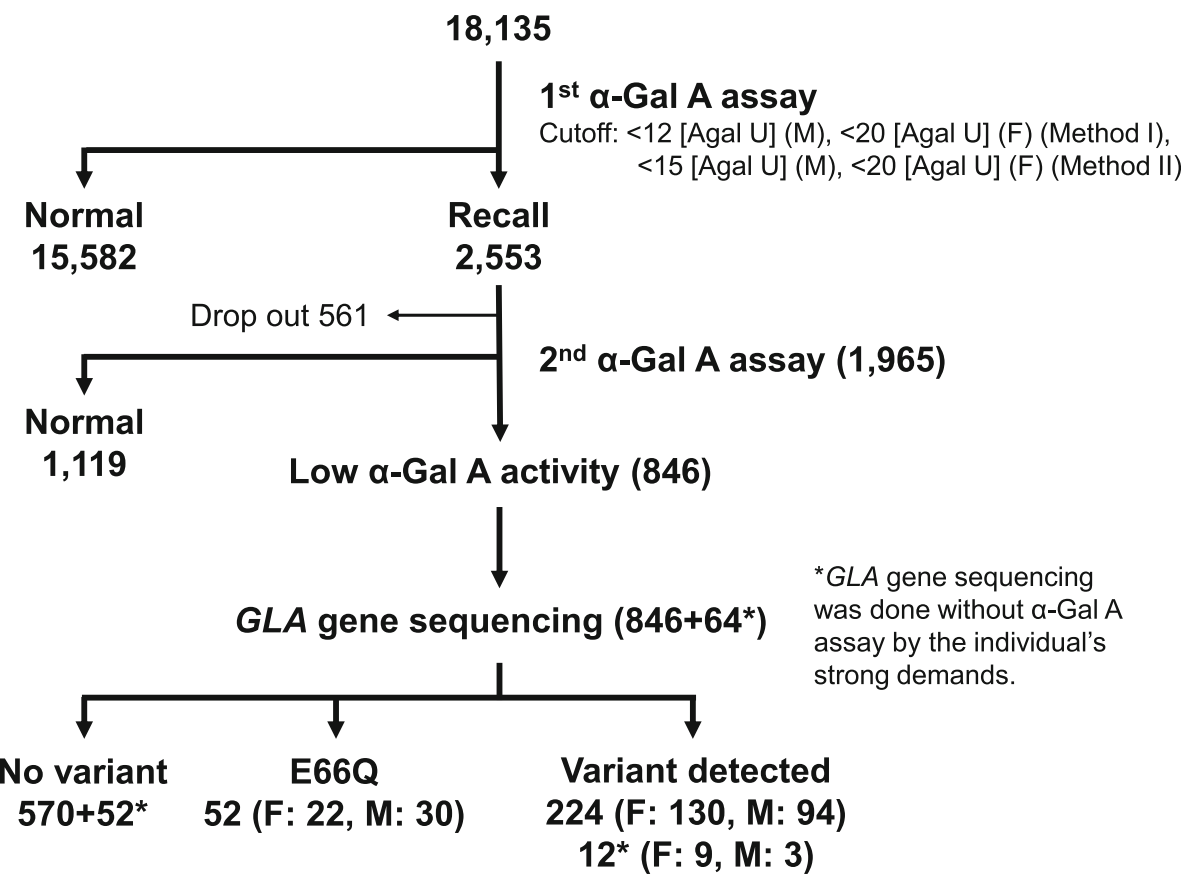

Fig. 1 Schematic representation of high-risk screening for Fabry disease 
A.

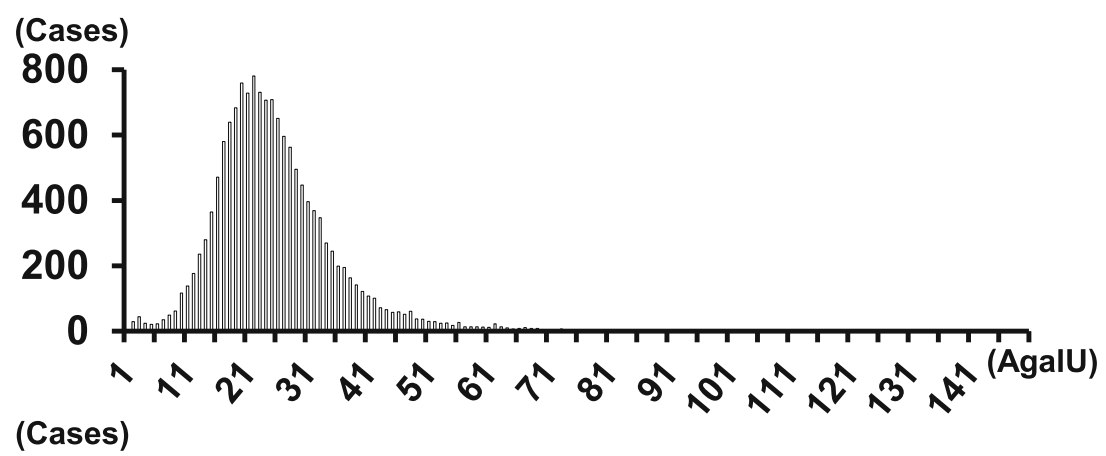

B.

(Cases)

C.
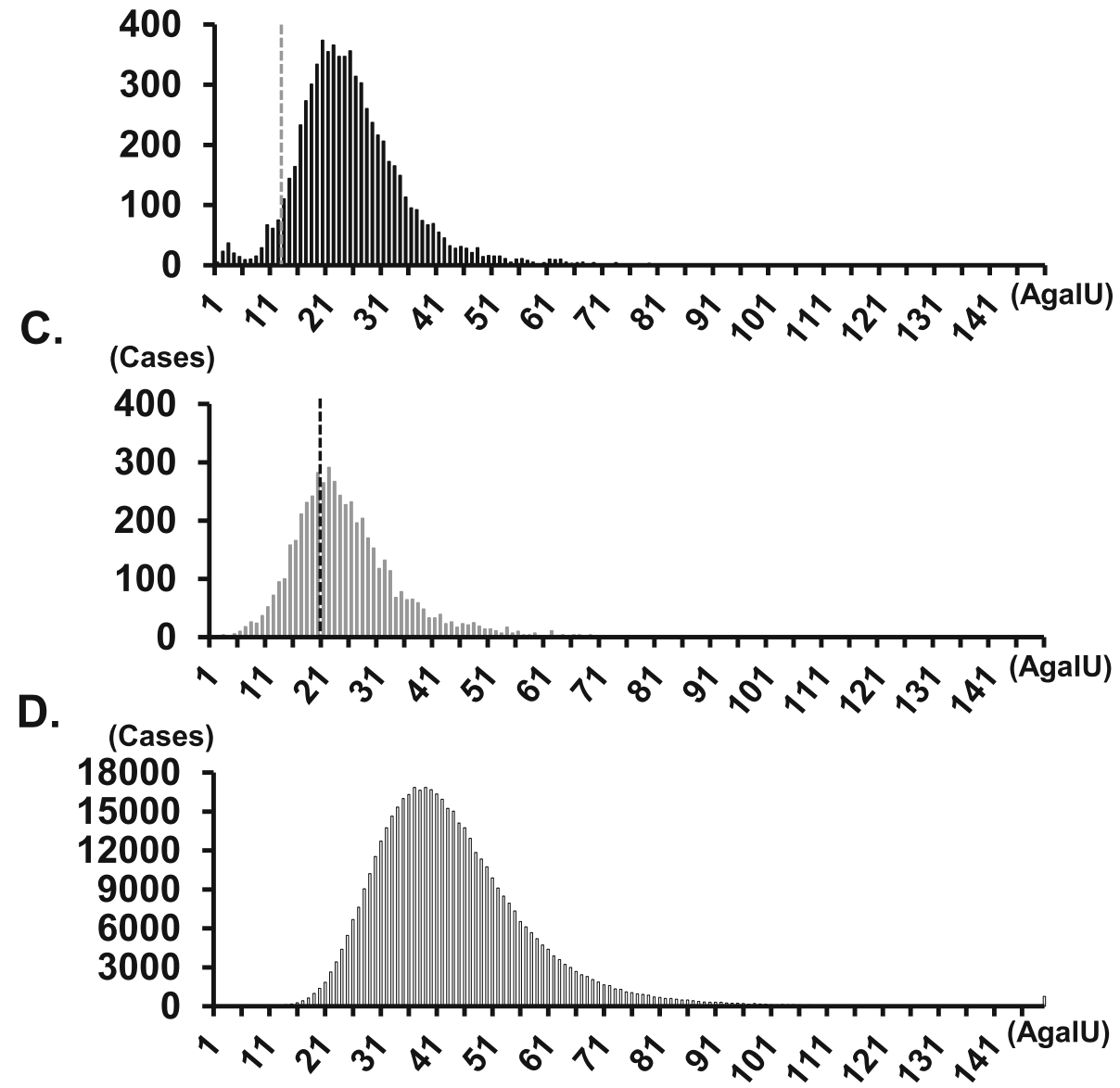

Fig. 2 Histograms of a-Gal A activity in the high-risk and newborn screening groups. Histograms of a-Gal A activity are shown for (a) the total population $(N=16,061)$, (b) men $(N=10,066)$, and $(\mathbf{c})$ women $(N=5558)$ in the high-risk screening group, as well as (d) the newborn screening group $(N=483,026)$. Dashed line: cutoff level

suitable for migalastat treatment (Table 2, Table S1 and Table S2). Regarding mutation type of the 101 variants, 64 were identified as missense mutations, 18 were frameshift mutations, 10 were nonsense mutations, 3 were in-frame deletions, 3 were intronic mutations, 2 were silent mutations (which potentially alter splicing), and 1 was a large deletion mutation of exon 3 and 4 . Of the 101 variants, 68 were registered in ClinVar or Fabrydatabase.org. Two variants, c.218C > A [18] and c.908_ 928del21 [17], were described in our previous report which included detailed information on each patient. The variant c. $625 \mathrm{~T}>\mathrm{C}$ was detailed in our previous report regarding NBS for FD [11]. Four variants, namely c. $725 \mathrm{~T}>\mathrm{C}$, c. $801+1 \mathrm{G}>\mathrm{A}$, c. $1124 \mathrm{G}>\mathrm{A}$, and c. $1165 \mathrm{C}>$ G, were reported by Tsukimura et al. [19], Li et al. [20], Iwafuchi et al. [21], and van der Tol et al. [22], respectively. The remaining 26 variants were considered novel variants. The most common variant was c.888G $>\mathrm{A} /$ p.M296I (allele frequency: 3.5\%, 5/143). The second most common variant was c.334C > T/p.R112C (2.8\%, 4/ 
Table 2 Variants of patients detected by high-risk screening for Fabry disease
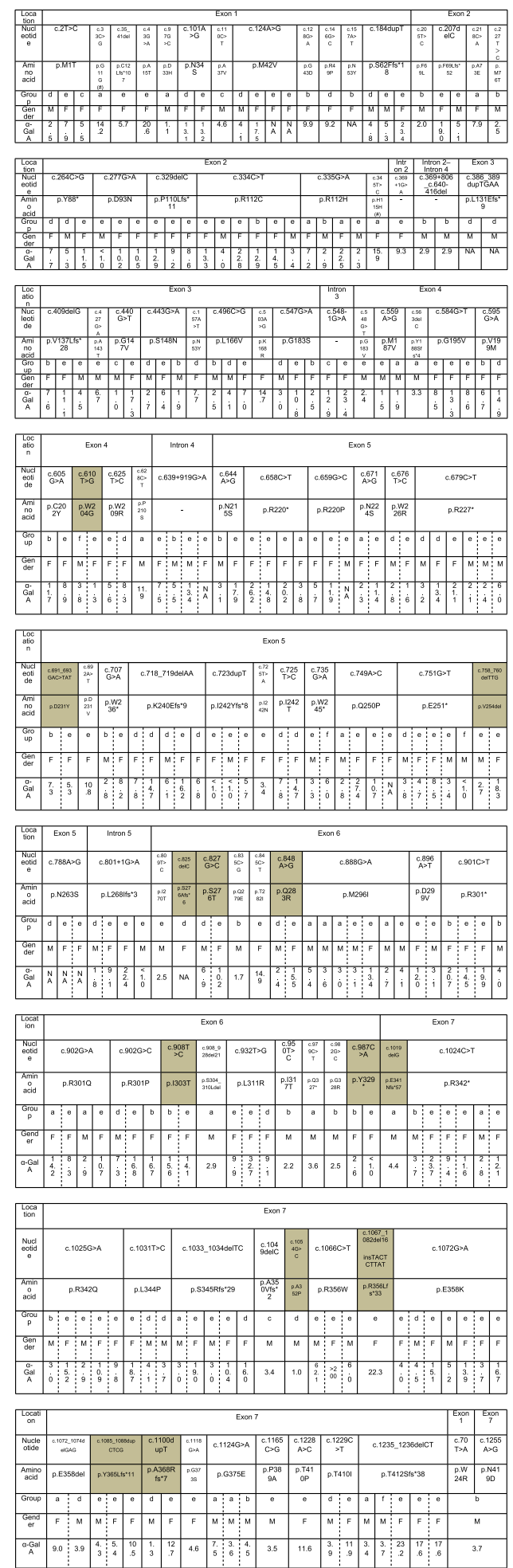

Variants of patients detected by high-risk screening for Fabry disease. a: Renal manifestations group. b: Cardiac manifestations group. c: Central neurological manifestations group. d: Peripheral neurological manifestations group. e: Family history group. f: "Other" group.

: novel variants
143). The third most common variants were c.335G $>\mathrm{A} /$ p.R112H (2.1\%, 3/143), c.658C > T/p.R220* $(2.1 \%, 3 /$ 143), c.679C > T/p.R227* (2.1\%, 3/143), c.718_719delAA/ p.K240Efs*8 (2.1\%, 3/143), c.902G > A/p.R301Q (2.1\%, 3/ 143), c.1033_1034delTC/p.S345Rfs*28 (2.1\%, 3/143), c.1124G > A/ p.G375E (2.1\%, 3/143), and c.1235 1236delCT/p.T412Sfs*37 (2.1\%, 3/143). The geographic distribution of the variants is illustrated in Fig. S2.

\section{Discussion}

High-risk screening for FD in selected patient cohorts has been reported. Doheny et al. [23] reanalyzed studies related to hemodialysis (27 reports, 23,954 men, and 12, 866 women), left ventricular hypertrophy (LVH) and/or hypertrophic cardiomyopathy (17 reports, 4054 men, and 1437 women), and ischemic or cryptogenic strokes (16 studies, 3904 men, and 2074 women). The revised prevalence was estimated as $0.21 \%$ for male and $0.15 \%$ for female hemodialysis patients, $0.94 \%$ for male and $0.90 \%$ for female cardiac patients, and $0.13 \%$ for male and $0.14 \%$ for female stroke patients. In the current study, the prevalence was estimated as $0.42 \%$ (male: $0.45 \%$, female: $0.38 \%$ ) in the renal manifestation group (a), $0.94 \%$ (male: $0.77 \%$, female: $1.33 \%$ ) in the cardiac manifestation group (b), and $0.22 \%$ (male: $0.20 \%$, female: $0.27 \%$ ) in the central neurological manifestation group (c), which are comparable to those of previous reports.

The prevalence of FD in the peripheral neurological manifestation group (d) was second highest at $4.37 \%$ (male: $4.98 \%$, female: $3.50 \%$ ), whereas the patients' age (male: median 13 [IQR: 11-18.5] years old, female: median 13 [IQR: 9-25] years old) was lesser than that of the other groups. Therefore, manifestations such as limb pain, acroparesthesia, clustered angiokeratoma, cornea verticillata, and hypo- or anhidrosis, could help identify FD patients. Politei et al. [24] has recommended that the cause of pain should be diagnosed early in unrecognized or newly diagnosed FD patients to improve treatment possibilities. FD experts consider that, regardless of sex or age, pain related to FD could be an early indication to commence ERT before potentially irreversible organ damage, to the kidneys, heart, or brain, prevails. However, a study conducted in Russia by NamazovaBaranova et al. [25] reported that no FD patients were identified from among 214 individuals (110 males and 104 females) with chronic limb pain. Moreover, the genetic, epidemiological, and ethnical information related to Russian FD patients are insufficient and future studies and information related to FD in Russia are required.

The prevalence of FD in individuals with a family history (e) was the highest at $23.40 \%$ (male: $12.49 \%$, female: $33.02 \%)$. GLA sequencing for individuals with a family history of FD was useful in identifying undiagnosed or pre-symptomatic FD patients. Therefore, when patients 
experience FD-related symptoms, clinicians should confirm the presence of a family history of FD and, if applicable, whether similar symptoms developed.

The variant spectra of GLA in Japanese patients have been reported [26, 27]. GLA gene analysis was previously performed for 207 FD patients [26]. The most common variant was c.888G > A/p.M296I (allele frequency: 5.8\%, 12/207). The second-most common variants were c.639+ 919G > A $(4.3 \%, 9 / 207)$ and c.679C > T/p.R227* (4.3\%, 9/207), followed by c.334C > T/p.R112C $(3.9 \%, 8 /$ 207), c.335G > A/p.R112H (3.9\%, 8/207), and c.902G > A/ p.R301Q $(3.9 \%, 8 / 207)$. In another study, 73 pathogenic variants were detected in 176 patients from 115 families [27] and the most common variant was c.334C > T/ p.R112C (allele frequency: $2.65 \%$ ). The second-most common variant was c.888G > A/p.M296I (1.89\%), followed by c.658C > T/p.R220* (1.52\%), c.718_719delAA/p.K240Efs"8 $(1.52 \%)$, and c.1025G > A/p.R342Q (1.52\%). The common variants identified in these studies, as well as those of the current study, overlapped.

We previously reported the first large-scale NBS program for FD in the western region of Japan [11]. A total of 599,711 newborns were screened and 26 GLA variants, including 8 novel variants, were detected in 57 neonates from 54 families. Of the 26 variants, 10 were also detected in the current study and most of them were detected in patients from western Japan (Fig. S1).

In the current study, 4 pedigrees $(4 / 68,5.9 \%)$ were perceived as de novo mutations (Table S3). The frequency might be comparable with those of previous studies performed in Japan (6.8\% (5/74); Kobayashi et al. [28]), Italy (2.8\% (3/108); Romani et al. [29]; and $14.3 \%$ (2/14); Morrone et al. [30]), Spain (4.5\% (1/22); Rodriguez-Mari et al. [31]), and the United Kingdom (6.3\% (1/16); Davies et al. [32]). A high frequency of de novo mutations has been reported in X-linked disorders, such as Duchenne muscular dystrophy (DMD) and hemophilia A (F8), and de novo mutations account for approximately one-third of the mutations in these two disorders [33, 34]. This is owing to lack of fitness to reproduce in the $\mathrm{X}$ chromosome. Hemizygous GLA mutations may have sufficient fitness to reproduce, reducing the frequency of de novo mutations [35]. Moreovrer, the presence of CpG dinucleotides also may increase mutational frequency [36]. GLA contains 19 CpG sites (1/68 bases) in the coding region compared to $F 8$ which has $68 \mathrm{CpG}$ sites (1/104 bases). Of the 19 potential mutation sites in GLA, 13 variants were identified in the current study, namely, c.146G $>\mathrm{C}$, c.334C > T, c.335G > A, c.427G > A, c.658C $>$ T, c.659G > C, c. $679 \mathrm{C}>\mathrm{T}, \quad$ c. $901 \mathrm{C}>\mathrm{T}, \quad$ c. $902 \mathrm{G}>\mathrm{A}, \quad$ c. $902 \mathrm{G}>\mathrm{C}$, c. $1024 \mathrm{C}>\mathrm{T}$, c. $1025 \mathrm{G}>\mathrm{A}$, and c.1066C $>$ T. Twenty-three variants were reported as de novo mutational hotspots (Table S3). However, no particular sites responsible for these de novo mutations were identified.
A few cases of homozygous or compound heterozygous female FD patients have been previously reported [30, 37]. However, homozygous or compound heterozygous female FD patients were not identified in the current study or in our previous NBS study [11]. In our NBS study, the frequency of male FD (the allele frequency of FD variants) was estimated to be 1:6212 $(0.016 \%)$, and the probability of homozygous female was extremely low as 1:38,588,944. Therefore, among female FD patients, only those with heterozygous GLA mutations are generally identified. Interestingly, a male patient harboring two GLA variants, c.70 T > A /p.W24R and c.1255A > G/p.N419D, was identified in the current study (Table S1). Unfortunately, genetic information regarding his family and his chromosome information were not available. It was unclear whether the two variants were in cis on a single $\mathrm{X}$ chromosome. Of $100 \mathrm{GLA}$ variants, 70 were detected only in single pedigrees, whereas 20 were identified in two pedigrees. Because a bias was introduced in the distribution of variants in these pedigrees, it was difficult to discuss the correlation between genotype and phenotype, especially organ specific pathogenicity.

On follow-up evaluation for each patient, 21 of the 26 novel variants were indicated as pathogenic, namely c.97G > C/p.D33H, c.157A > T/p.N53Y, c.184dupT/p.S62 Ffs"18, c.205 T > C/p.F69L, c.207del/p.F69Lfs"52, c.264C > G/p.Y88*, c.329del/p.P110Lfs"11, c.386_389dupTGAA/ p.L131Efs"9, c.440G > T/p.G147V, c.563delC/p.Y188Sfs*4, c.610 T > G/p.W204G, c.691_693GAC > TAT/p.D231Y, c.8 25delC/p.S276Afs*6, c.827G > C/p.S276T, c.848A > G/ p.Q283R, c.908 T > C/p.I303T, c.987C > A/p.Y329*, c.101 9delG/p.E341Nfs*57, c.1054G > C/p.A352P, c.1085_1088 dupCTCG/p.Y365Lfs*11, and c.1100dupT/p.A368Rfs*7. Furthermore, five variants were identified herein, which were not registered in the ClinVar or Fabry-database.org, specifically c.725 T > C/p.I242T, c.801 + 1G > A/p.L268If**3, c.908_923del21/p.S304_310Ldel, c.1124G > A/p.G375E, and c.1165C > G/p.P389A. Patients harboring the aforementioned variants developed FD-related symptoms, and some had even died of stroke or cardiac failure.

Even during high-risk FD screening, individuals are assigned an uncertain diagnosis in the absence of classical FD symptoms and when variants of unknown significance (VOUS) in the GLA gene are identified. This leads to a risk of misdiagnosis, inappropriate counseling, and extremely costly treatment. Therefore, numerous studies have attempted to generate a diagnostic algorithm for FD, which maximally excludes these risks [38]. In our high-risk screening, individuals presenting decreased activity (<cutoff levels) with known pathogenic variants, classical signs or symptoms of FD, or a family history of FD were definitely diagnosed with FD. However, among individuals presenting decreased activity $(<$ 
cutoff levels) with VOUS and late onset signs or symptoms such as cryptogenic stroke, proteinuria, or LVH without classic type signs or symptoms, a definite diagnosis is difficult to achieve. Moreover, because the disease state during late-onset FD is potentially not improved through ERT [39], the therapeutic effect of ERT does not facilitate the diagnosis of FD. Blood LysoGb3 assays and tissue diagnosis in a myocardial or renal biopsy may be sufficient for a definite diagnosis of FD [40]. Moreover, analysis using iPSC technology, such as Gb3 accumulation in iPSC-derived vascular endothelial cells, may lead to a definite diagnosis [41].

High-risk FD screening has a potential for falsepositive findings. Figure $2 \mathrm{a}, \mathrm{b}$, and $\mathrm{c}$ show the histograms of this high-risk screening for all individuals, men, and women, respectively, in Method I. The median $\alpha-$ Gal A activity was 24.47, 24.50, and 24.06 (AgalU) among all individuals, men, and women, respectively. A dotted line indicates the cutoff value: < 12 [AgalU] for men and $<20$ [AgalU] for women; 50\% of median $\alpha-G a l$ A activity for men and $80 \%$ of median $\alpha$-Gal A activity for women. Heterozygous female patients have an almost normal range of $\alpha$-Gal A activity, resulting in falsenegative findings in screening studies. Linthorst et al. [42] reported that $40 \%(16 / 40)$ of female patients with FD are not identified, considering a cutoff $<50 \%$ of the normal control. Although we used a higher cutoff $<80 \%$ of median $\alpha$-Gal A activity, false-negative findings may have been obtained among the female FD patients herein. Additional tests, such as blood Lyso-Gb3 assays [43], hotspot mutation screening [44], or even whole GLA gene sequencing, may improve the rate of falsenegative results. Most patients with FD in Taiwan harbor variants out of a pool of only 21 pathogenic mutations [45]. Therefore, in regions such as Taiwan, where hotspot mutations can be detected, hotspot mutation screening is effective for high-risk screening among women. In regions such as Japan, where hotspot mutations cannot be detected and several variants are found, hotspot mutation screening is not as effective. Whole GLA gene sequencing is difficult to perform among all female patients included in the high-risk screening group because of cost-balance issues. The assay of LysoGb3 in dried blood spots (DBSs) is considered an effective and realistic alternative for high-risk screening among women [43]. We will consider applying the LysoGb3 assay for high-risk screening in future studies.

Figure $2 \mathrm{~d}$ shows a histogram of $\alpha$-Gal A activity in an NBS study in Method I [11]. The median $\alpha$-Gal A activity among neonates was 42.58 (AgalU), which is approximately 2-fold that of the current high-risk screening populations. FD is associated with a significantly reduced life expectancy compared to that of the general population [45]. Although the detailed mechanism for the low
$\alpha$-Gal A activity in adults is unknown, it may be associated with a premature aging process through the dysfunction of blood vessels. Therefore, aging and low $\alpha$ Gal A activity are closely related.

The cutoff values in the high-risk screening populations were 12 (AgalU) for men and 20 (AgalU) for women, which is representative of the cutoff values for the 0.5 percentile in the NBS population. This is because $\alpha-G a l$ A activity in adults is lower than that of neonates. The current high-risk screening program identified individuals who are considered suitable candidates for migalastat treatment. Some patients were already receiving migalastat treatment. Moreover, gene therapy holds promise in effectively treating various diseases, and the clinical trials for gene therapy for FD are ongoing in Canada and the USA (https://fabrydiseasenews.com/ gene-therapy-for-fabry-disease/). In the future, the development of new treatment methods for FD, other than ERT, is expected.

\section{Conclusions}

In the current study, we performed high-risk screening for FD in individuals from all the prefectures in Japan. A total of 18,199 individuals were screened using DBSs, and 101 GLA variants, including 26 novel variants, were identified among 236 patients from 143 families. The distribution of variants is diverse for each region of Japan, and de novo mutations in the GLA gene were detected in a significant proportion of these variants. Therefore, further novel mutations would likely be identified in the future. Regarding treatment, $33 \%$ of the FD patients were identified as suitable candidates for migalastat therapy and $39 \%$ of the GLA variants were identified as amenable. Therefore, the simple screening protocol using DBSs could be useful in the early diagnosis and selection of appropriate treatments of FD in high-risk and undiagnosed patients with various renal, cardiac, or neurological manifestations. FD screening is essential for individuals presenting with peripheral neuropathy or a family history of FD as both have been identified as strong predictive factors in FD development.

\section{Methods \\ Study design}

To identify FD patients by performing high-risk screening in 18,135 individuals, enrolled from October 2006 to March 2019, with renal, cardiac, or neurological manifestations from all the prefectures in Japan; the following study design was implemented. A total of 601 hospitals, from all the prefectures in Japan, participated in this study. From October 2006 to March 2019, the DBSs of 18,135 patients with various cardiac, renal, or neurological manifestations were assessed. Written informed consent was obtained from the patients or their parents 
(in cases where the patients were not of legal age). The individuals were enrolled in the study if they developed at least one of the following manifestations: (a) renal manifestations, such as proteinuria, chronic kidney disease, diabetic nephropathy, mulberries in the urine, and the need for dialysis; (b) cardiac manifestations, such as LVH detected using electrocardiography or echocardiography; (c) central neurological manifestations, such as parkinsonism, hearing loss, and history of stroke; (d) peripheral neurological manifestations, including limb pain, acroparesthesia, clustered angiokeratoma, cornea verticillata, and hypo-anhidrosis; (e) family history of FD; or (f) other reasons, such as liver failure and unavailable information.

DBS specimens were prepared as reported previously [17]. Briefly, after dropping blood spots onto filter papers (Toyo Roshi Kaisha, Ltd., Tokyo, Japan), the DBSs were dried for at least $4 \mathrm{~h}$ at room temperature, sent to Kumamoto University by mail within 1 week of preparation, and if necessary, stored at $-20^{\circ} \mathrm{C}$ until use. The high-risk screening for FD using $\alpha-G a l$ A assays with DBSs was performed in two steps. In the first step, individuals with $\alpha$-Gal A activity under the cutoff value (in Method I: < 12 [AgalU] for males and $<20$ [AgalU] for women; and Method II: < 15 [AgalU] for males and $<20$ [AgalU] for women) were recalled and their DBSs reprepared. In the second step, individuals with $\alpha$-Gal A activity under the cutoff value were assessed clinically, and GLA gene sequencing was performed after informed consent was obtained from the patients or their parents (in cases where the patients were not of legal age).

\section{a-Gal a assay \\ Method I}

$\alpha$-Gal A assays were performed as described previously [11]. Briefly, a single $3.2 \mathrm{~mm}$ diameter disk, punched from DBSs, was incubated in a well of a 96-well clear microwell plate (Corning, NY, USA) with $40 \mu \mathrm{L}$ of McIlvaine buffer $\left(100 \mathrm{mM}\right.$ citrate; $200 \mathrm{mM} \mathrm{NaH} \mathrm{PO}_{4}$; 36.8: 63.2; $\mathrm{pH} 6.0$ ) and processed for extraction at room temperature for $2 \mathrm{~h}$. Aliquots of $30 \mu \mathrm{L}$ blood extract were transferred to fresh 96-microwell plates. An aliquot of $100 \mu \mathrm{L}$ of the reaction mixture $(3.5 \mathrm{mM} 4$ methylumbelliferyl- $\alpha$-D-galactopyranoside (4MU- $\alpha \mathrm{Gal})$; $100 \mathrm{mM}$ citrate; $200 \mathrm{mM} \mathrm{K} \mathrm{HPO}_{4} ; 100 \mathrm{mM} \mathrm{N}$-acetyl-Dgalactosamine) was added to each well of the microwell plates and incubated at $37^{\circ} \mathrm{C}$ for $24 \mathrm{~h}$. The reaction was terminated using $150 \mu \mathrm{L}$ of termination solution (300 $\mathrm{mM}$ glycine/NaOH; $\mathrm{pH}$ 10.6) immediately after the reaction occurred. The fluorescence intensity, from the 4methylumbelliferones in the wells, was measured at 450 $\mathrm{nm}$ using a fluorescence plate reader (BIO-TEK, Winooski, VT, USA). One unit (1 AgalU) of enzymatic activity was equal to $0.34 \mathrm{pmol}$ of $4 \mathrm{MU}-\alpha \mathrm{Gal}$ cleaved/h per disk.

\section{Method II}

Method II for multiple assays was developed in collaboration with KM Biologics Co., Ltd. (see details at JP6360848B) and practically implemented from November 2016. Briefly, a single $3.2 \mathrm{~mm}$ diameter disk, punched from DBSs, was incubated in a well of a 96well clear microwell plate (AS ONE Corporation, Osaka, Japan) with $100 \mu \mathrm{L}$ of $25 \mathrm{mM}$ citrate/potassium phosphate buffer ( $\mathrm{pH} 6.0$ ) containing $5 \mathrm{mM} \mathrm{MgCl}_{2}, 0.5 \mathrm{mM}$ DTT, $0.05 \% \mathrm{NaN}_{3}$, and $0.1 \%$ Triton $\mathrm{X}-100$ for $1 \mathrm{~h}$ at room temperature with gentle mixing. A $20-\mu \mathrm{L}$ aliquot of the extract was then added to $40 \mu \mathrm{L}$ of the reaction mixture (3.0 mM 4MU- $\alpha$ Gal; $100 \mathrm{mM} \mathrm{N}$-acetyl-D-galactosamine in $100 \mathrm{mM}$ citrate $/ 200 \mathrm{mM}$ potassium phosphate buffer; $\mathrm{pH} 4.4$ ) in a black 96-well microwell plate (Thermo Fisher Scientific Inc., MA, USA). The reaction mixture was incubated at $38^{\circ} \mathrm{C}$ for $3 \mathrm{~h}$, and the reaction was terminated with $200 \mu \mathrm{L}$ of $300 \mathrm{mM}$ glycine $/ \mathrm{NaOH}$ buffer ( $\mathrm{pH}$ 10.6) containing $10 \mathrm{mM}$ ethylenediaminetetraacetic acid (EDTA) to measure fluorescence intensity. The residual extract could be used for the assay of acid $\alpha$-glucosidase (Pompe disease) and glucocerebrosidase (Gaucher disease) activity.

\section{Sequencing of the GLA gene Sanger sequencing}

Genomic DNA was extracted from total blood using a Gentra Puregene Blood Kit (Qiagen, Hilden, Germany), or equivalent product, and stored at $-80^{\circ} \mathrm{C}$ until use. All seven exons and the flanking intronic sequences of the GLA gene were amplified using PCR as described previously $[46,47]$. The region of intron 4 was also amplified to evaluate the variant, c.639+919G >A [48]. The PCR products were sequenced using an $\mathrm{ABI} 3500 \mathrm{xl}$ autosequencer (Applied Biosystems) and analyzed using Sequencher 5.0 (Gene Codes Corporation, Ann Arbor, MI, USA).

\section{Next-generation sequencing (NGS)}

A high-throughput NGS assay for GLA genewas developed in collaboration with KM Biologics Co., Ltd. and practically implemented from September 2017; the protocol is described in our previous report [11]. Briefly, the 13.3-kb region, including GLA, was amplified using long-range PCR. Library preparation and sequencing were performed using a Nextera XT Kit (Illumina, San Diego, CA, USA) and MiSeq sequencer (Illumina). After sequencing runs were completed, the data were aligned with those of the human reference genome sequence (NC_000023.10) using MiSeq Reporter software (Illumina). Sequence data analysis, mapping, and variant calling were streamlined using MiSeq Reporter v2 (Illumina). Sequencing reads were visualized using IGV_2.3.10 (Broad Institute). Variants detected in the GLA gene by NGS were resequenced using the Sanger method. 


\section{Prediction and statistical tools}

\section{Significance analysis for the variants}

The GLA mRNA reference sequence (RefSeq; NM 000169.2) was used in this study, whereby the " $A$ " nucleotide of the ATG codon at nucleotide position 111 of RefSeq constituted +1 numbering of the cDNA sequence. The ATG codon also represented +1 for the amino acid numbering as set forth by the $\alpha$-Gal A preprotein sequence NP_000160.1. Variant nomenclature followed the guidelines established by the Human Genome Variation Society (http://varnomen.hgvs.org/). Public databases, including Fabry-database.org [1] (http:// fabry-database.org/, updated on February 15, 2019), and ClinVar [2] (http://www.ncbi.nlm.nih.gov/clinvar) were used to classify each variant. The software PolyPhen-2 [49] (http://genetics.bwh.harvard.edu/pph2) was used for missense mutations to predict the potential impact of an amino acid alteration on $\alpha$-Gal A function. The amenability of each variant for the pharmacological chaperone migalastat was confirmed using the website of Amicus Therapeutics, Inc. (http://www.galafoldamenabilitytable. jp/reference).

\section{Supplementary information}

Supplementary information accompanies this paper at https://doi.org/10. 1186/s13023-020-01494-6.

Additional file 1. (Microsoft PowerPoint Presentation.pptx): Figure S1. Histograms of a-Gal A activity in the high-risk population. A. Renal manifestations group (total, $N=8004$; male, $N=4884$; and Female, $N=2905$ ). B. Cardiac manifestations group (total, $N=2410$; male, $N=1735$; and female, $N=651$ ). C. Central neurological manifestations (total, $N=2593$; male, $N=1609$; and female, $N=803$ ). D. Peripheral neurological manifestations group (total, $N=316$; male, $N=181$; and female, $N=134$ ). E. Family history group (total, $N=334$; male, $N=141$; and female, $N=190$ ).

Additional file 2. (Microsoft PowerPoint Presentation.pptx): Figure S2. Geographic distribution of the variants detected from high-risk screening for Fabry disease in Japan

Additional file 3: Table S1. Demographic characteristics of patients identified by high-risk screening for Fabry disease. NA: not available, (\#): Potential alteration of splicing predicted by Human Splicing Finder. Amenability; +: amenable, -: not amenable,?: unknown (http://www.galafoldamenabilitytable.jp/reference, access at 2020/01/06). p.F69L/c.207C > A was reported by Umeda et al., Hum. Genome Var. 2 (2015) 15044. Gray box: novel variants

Additional file 4: Table S2. Variants detected during high-risk screening for Fabry disease. Fabry-database.org: last updated 2019/02/15 ver.3.2.2. NR: not registered, (\#): Potential alteration of splicing predicted by Human Splicing Finder. ${ }^{* *}+$ : amenable, - : not amenable,?: unknown, NA: not available, http://www.galafoldamenabilitytable.jp/reference (access at 2020/01/06). p.F69L/c.207C > A was reported by Umeda et al., Hum. Genome Var. 2 (2015) 15044. Gray box: novel variants

Additional file 5: Table S3. De novo mutations detected in patients with Fabry disease.

\section{Abbreviations}

DBSs: Dried blood spots; DMD: Duchenne muscular dystrophy; ERT: Enzyme replacement therapy; FD: Fabry disease; NBS: Newborn screening; LVH: Left ventricular hypertrophy; NGS: Next-generation sequencing; a-Gal A: agalactosidase A; 4MU-aGal: 4-methylumbelliferyl-a-D-galactopyranoside

\section{Acknowledgements}

We are grateful to Ms. Fumiko Nozaki, Ms. Naomi Yano, Ms. Ayuko Tateishi, Ms. Emi Harakawa, Ms. Yasuyo Sakamoto, Ms. Hiroko Nasu, and Ms. Matsumi Harada for providing technical support related to this study.

\section{Authors' contributions}

SY, JK, KS, and KN were responsible for the design of the research. SY, JK, TS, KM, KS, SM, and FE contributed to measurements and data collection. SY, JK, TS, KM, SM, and FE checked and analyzed the data. JK and KS wrote the manuscript. JK and KN supervised this study. The authors read and approved the final manuscript for submission. All authors have agreed both to be personally accountable for the author's own contributions and to ensure that questions related to the accuracy or integrity of any part of the work, even ones in which the author was not personally involved, are appropriately investigated, resolved, and the resolution documented in the literature.

\section{Funding}

This study was supported in part by a Health, Labour and Welfare Sciences Research Grant for Research on Rare and Intractable Diseases, Ministry of Health, Labour and Welfare, Japan; a Grant-in-Aid for Pediatric Research from the Ministry of Health, Labour and Welfare, Japan; a Grant-in-Aid from the Japan Agency for Medical Research and Development; and a Grant-in-Aid for Scientific Research from the Ministry of Education, Culture, Sports, Science, and Technology, Japan.

\section{Availability of data and materials}

The datasets used and/or analyzed during the current study are available from the corresponding author on reasonable request.

\section{Ethics approval and consent to participate}

This study was approved by the Kumamoto University Ethics Committee. Written informed consent was obtained from the patients or their parents (in cases where the patients were not of legal age).

\section{Consent for publication}

Not applicable.

\section{Competing interests}

The authors declare that they have no competing interests.

\section{Author details}

${ }^{1}$ Department of Pediatrics, Graduate School of Medical Sciences, Kumamoto University, 1-1-1 Honjo, Chuo-ku, Kumamoto City, Kumamoto 860-8556, Japan. ${ }^{2}$ KM Biologics Co., Ltd., Kumamoto, Japan. ${ }^{3}$ Kumamoto-Ezuko Medical Center for Disabled Children, Kumamoto, Japan.

Received: 4 March 2020 Accepted: 5 August 2020

Published online: 26 August 2020

References

1. Saito S, Ohno K, Sakuraba H. Fabry-database.Org: database of the clinical phenotypes, genotypes and mutant a-galactosidase a structures in Fabry disease. J Hum Genet. 2011. https://doi.org/10.1038/jhg.2011.31.

2. Landrum MJ, Lee JM, Benson M, Brown G, Chao C, Chitipiralla S, et al. ClinVar: public archive of interpretations of clinically relevant variants. Nucleic Acids Res. 2016. https://doi.org/10.1093/nar/gkv1222.

3. Desnick RJ, loannou YA, Eng CM. Alpha-galactosidase a deficiency: Fabry disease. In: Scriver CR, Beaudet AL, Sly WS, Valle D, editors. The metabolic and molecular bases of inherited disease. 8th ed. New York: McGraw-Hill; 2001. p. 3733-74.

4. Mehta A, Beck M, Eyskens F, Feliciani C, Kantola I, Ramaswami U, et al. Fabry disease: a review of current management strategies. QJM. 2010. https://doi. org/10.1093/qjmed/hcq117.

5. Nakamura K, Hattori K, Endo F. Newborn screening for lysosomal storage disorders. Am J Med Genet C Semin Med Genet. 2011. https://doi.org/10. 1002/ajmg.c.30291.

6. Ramaswami U, Whybra C, Parini R, Pintos-Morell G, Mehta A, SunderPlassmann G, et al. Clinical manifestations of Fabry disease in children: data from the Fabry outcome survey. Acta Paediatr. 2006. https://doi.org/10. 1080/08035250500275022. 
7. Eng CM, Fletcher J, Wilcox WR, Waldek S, Scott CR, Sillence DO, et al. Fabry disease: baseline medical characteristics of a cohort of 1765 males and females in the Fabry registry. J Inherit Metab Dis. 2007. https://doi.org/10. 1007/s10545-007-0521-2.

8. MacDermot KD, Holmes A, Miners AH. Anderson-Fabry disease: clinical manifestations and impact of disease in a cohort of 60 obligate carrier females. J Med Genet. 2001. https://doi.org/10.1136/jmg.38.11.769.

9. Marchesoni CL, Roa N, Pardal AM, Neumann P, Caceres G, Martinez P, et al. Misdiagnosis in Fabry disease. J Pediatr. 2010. https://doi.org/10.1016/j.jpeds. 2010.02.012.

10. Terryn W, Cochat P, Froissart R, Ortiz A, Pirson Y, Poppe B, et al. Fabry nephropathy: indications for screening and guidance for diagnosis and treatment by the European renal best practice. Nephrol Dial Transplant. 2013. https://doi.org/10.1093/ndt/gfs526.

11. Sawada T, Kido J, Yoshida S, Sugawara K, Momosaki K, Inoue T, et al. Newborn screening for Fabry disease in the western region of Japan. Mol Genet Metab Rep. 2020. https://doi.org/10.1016/j.ymgmr.2019.100562.

12. Liao HC, Hsu TR, Young L, Chiang CC, Huang CK, Liu HC, et al. Functional and biological studies of a-galactosidase a variants with uncertain significance from newborn screening in Taiwan. Mol Genet Metab. 2018. https://doi.org/10.1016/j.ymgme.2017.06.002.

13. Burton BK, Charrow J, Hoganson GE, Waggoner D, Tinkle B, Braddock SR, et al. Newborn screening for lysosomal storage disorders in Illinois: the initial 15-month experience. J Pediatr. 2017. https://doi.org/10.1016/j.jpeds. 2017.06.048

14. Germain DP, Hughes DA, Nicholls K, Bichet DG, Giugliani R, Wilcox WR, et al. Treatment of Fabry's disease with the pharmacologic chaperone Migalastat. N Engl J Med. 2016. https://doi.org/10.1056/NEJMoa1510198.

15. Beck M, Hughes D, Kampmann C, Larroque S, Mehta A, Pintos-Morell G, et al. Long-term effectiveness of agalsidase alfa enzyme replacement in Fabry disease: a Fabry outcome survey analysis. Mol Genet Metab Rep. 2015. https://doi.org/10.1016/j.ymgmr.2015.02.002.

16. Hughes DA, Nicholls K, Shankar SP, Sunder-Plassmann G, Koeller D, Nedd K, et al. Oral pharmacological chaperone migalastat compared with enzyme replacement therapy in Fabry disease: 18-month results from the randomised phase III ATTRACT study. J Med Genet. 2017. https://doi.org/10. 1136/jmedgenet-2016-104178.

17. Nakagawa N, Sawada J, Sakamoto N, Takeuchi T, Takahashi F, Maruyama J, et al. High-risk screening for Anderson-Fabry disease in patients with cardiac, renal, or neurological manifestations. J Hum Genet. 2019. https:// doi.org/10.1038/s10038-019-0633-1.

18. Nishino T, Obata Y, Furusu A, Hirose M, Shinzato K, Hattori K, et al. Identification of a novel mutation and prevalence study for fabry disease in Japanese dialysis patients. Ren Fail. 2012. https://doi.org/10.3109/0886022X. 2012.669300.

19. Tsukimura T, Nakano S, Togawa T, Tanaka T, Saito S, Ohno K, et al. Plasma mutant a-galactosidase a protein and globotriaosylsphingosine level in Fabry disease. Mol Genet Metab Rep. 2014. https://doi.org/10.1016/j.ymgmr. 2014.07.005

20. Li P, Zhang L, Zhao N, Xiong Q, Zhou Y-A, Wu C, et al. A novel a -galactosidase a splicing mutation predisposes to Fabry disease. Front Genet. 2019. https://doi.org/10.3389/fgene.2019.00060.

21. Iwafuchi $Y$, Maruyama H, Morioka T, Noda S, Nagata H, Oyama Y, et al. Enzyme replacement therapy in a patient of heterozygous Fabry disease: clinical and pathological evaluations by repeat kidney biopsy and a successful pregnancy. CEN Case Rep. 2017. https://doi.org/10.1007/s13730017-0277-y.

22. van der Tol L, Verhamme C, van Schaik IN, van der Kooi AJ, Hollak CEM, Biegstraaten $\mathrm{M}$. In patients with an a-galactosidase a variant, small nerve fibre assessment cannot confirm a diagnosis of Fabry disease. Jimd Rep. 2016. https://doi.org/10.1007/8904_2015_503.

23. Doheny D, Srinivasan R, Pagant S, Chen B, Yasuda M, Desnick RJ. Fabry disease: prevalence of affected males and heterozygotes with pathogenic GLA mutations identified by screening renal, cardiac and stroke clinics, 1995-2017. J Med Genet. 2018. https://doi.org/10.1136/jmedgenet-2017-105080.

24. Politei JM, Bouhassira D, Germain DP, Goizet C, Guerrero-Sola A, Hilz MJ, et al. Pain in Fabry disease: practical recommendations for diagnosis and treatment. CNS Neurosci Ther. 2016. https://doi.org/10.1111/cns.12542.

25. Namazova-Baranova LS, Baranov AA, Pushkov AA, Savostyanov KV. Fabry disease in children: a federal screening programme in Russia. Eur J Pediatr. 2017. https://doi.org/10.1007/s00431-017-2992-y.
26. Sakuraba H, Tsukimura T, Togawa T, Tanaka T, Ohtsuka T, Sato A, et al. Fabry disease in a Japanese population-molecular and biochemical characteristics. Mol Genet Metab Rep. 2018. https://doi.org/10.1016/j.ymgmr.2018.10.004.

27. Kobayashi M, Ohashi T, Kaneshiro E, Higuchi T, Ida H. Mutation spectrum of a-galactosidase gene in Japanese patients with Fabry disease. J Hum Genet. 2019. https://doi.org/10.1038/s10038-019-0599-z.

28. Kobayashi M, Ohashi T, lizuka S, Kaneshiro E, Higuchi T, Eto Y, et al. Frequency of de novo mutations in Japanese patients with Fabry disease. Mol Genet Metab Rep. 2014. https://doi.org/10.1016/2Fj.ymgmr.2014.07.001.

29. Romani I, Borsini W, Nencini P, Morrone A, Ferri L, Frusconi S, et al. De novo diagnosis of Fabry disease among Italian adults with acute ischemic stroke or transient ischemic attack. J Stroke Cerebrovasc Dis. 2015. https://doi.org/ 10.1016/j.jstrokecerebrovasdis.2015.07.012.

30. Morrone A, Cavicchi C, Bardelli T, Antuzzi D, Parini R, Rocco MD, et al. Fabry disease: molecular studies in Italian patients and $\mathrm{X}$ inactivation analysis in manifesting carriers. J Med Genet. 2003. https://doi.org/10.1136/jmg.40.8. e103.

31. Rodriguez-Mari A, Coll MJ, Chabas A. Molecular analysis in Fabry disease in Spain: fifteen novel GLA mutations and identification of a homozygous female. Hum Mutat. 2003. https://doi.org/10.1002/humu.9172.

32. Davies JP, Eng CM, Hill JA, Malcolm S, MacDermot $K$, Winchester $B$, et al. Fabry disease: fourteen alpha-galactosidase a mutations in unrelated families from the United Kingdom and other European countries. Eur J Hum Genet. 1996. https://doi.org/10.1159/000472202.

33. Kong X, Zhong X, Liu L, Cui S, Yang Y, Kong L. Genetic analysis of 1051 Chinese families with Duchenne/Becker muscular dystrophy. BMC Med Genet. 2019. https://doi.org/10.1186/s12881-019-0873-0.

34. Beaudet AL, Scriver CR, Sly WS, Valle D. Genetics, biochemistry and molecular bases of variant human phenotypes. In: Scriver CR, Beaudet AL, Sly WS, Valle D, editors. The metabolic and molecular bases of inherited disease. 8th ed. New York: McGraw-Hill; 2001. p. 3-45.

35. Haldane JB. The rate of spontaneous mutation of a human gene. J Genet. 1935:2004. https://doi.org/10.1007/BF02717892.

36. Cooper DN, Youssoufian $\mathrm{H}$. The CpG dinucleotide and human genetic disease. Hum Genet. 1988. https://doi.org/10.1007/bf00278187.

37. Oder D, Vergho D, Ertl G, Wanner C, Nordbeck P. Case report of a 45-year old female Fabry disease patient carrying two alpha-galactosidase a gene mutation alleles. BMC Med Genet. 2016. https://doi.org/10.1186/s12881-0160309-z.

38. Smid BE, van der Tol L, Cecchi F, Elliott PM, Hughes DA, Linthorst GE, et al. Uncertain diagnosis of Fabry disease: consensus recommendation on diagnosis in adults with left ventricular hypertrophy and genetic variants of unknown significance. Int J Cardiol. 2014. https://doi.org/10.1016/j.jicard. 2014.09.001.

39. Sasa H, Nagao M, Kino K. Safety and effectiveness of enzyme replacement therapy with agalsidase alfa in patients with Fabry disease: post-marketing surveillance in Japan. Mol Genet Metab. 2019. https://doi.org/10.1016/j. ymgme.2019.02.005

40. Thurberg BL, Fallon JT, Mitchell R, Aretz T, Gordon RE, O'Callaghan MW. Cardiac microvascular pathology in Fabry disease: evaluation of endomyocardial biopsies before and after enzyme replacement therapy. Circulation. 2009. https://doi.org/10.1161/CIRCULATIONAHA.108.841494.

41. Tseng WL, Chou SJ, Chiang HC, Wang ML, Chien CS, Chen KH, et al. Imbalanced production of reactive oxygen species and mitochondrial antioxidant SOD2 in Fabry disease-specific human induced pluripotent stem cell-differentiated vascular endothelial cells. Cell Transplant. 2017. https:// doi.org/10.3727/096368916X694265.

42. Linthorst GE, Poorthuis BJHM, Hollak CEM. Enzyme activity for determination of presence of Fabry disease in women results in $40 \%$ false-negative results. J Am Coll Cardol. 2008. https://doi.org/10.1016/j.jacc.2008.02.050.

43. Duro G, Zizzo C, Cammarata G, Burlina A, Burlina A, Polo G, et al. Mutations in the GLA gene and LysoGb3: is it really Anderson-Fabry disease? Int J Mol Sci. 2018. https://doi.org/10.3390/ijms19123726.

44. Lu Y-H, Huang P-H, Wang L-Y, Hsu T-R, Li H-Y, Lee P-C, et al. Improvement in the sensitivity of newborn screening for Fabry disease amaong females through the use of a high-throughput and cost-effective methods, DNA mass spectrometry. J Hum Genet. 2018. https://doi.org/10.1038/s10038-0170366-y.

45. Waldek S, Patel MR, Banikazemi M, Lemay R, Lee P. Life expectancy and case of death in males and females with Fabry disease: findings from the Fabry registry. Genet Med. 2009. https://doi.org/10.1097/GIM.0b013e3181 bb05bb. 
46. Blanch LC, Meaney C, Morris CP. A sensitive mutation screening strategy for Fabry disease: detection of nine mutations in the a-galactosidase a gene. Hum Mutat. 1996. https://doi.org/10.1002/(SICI)1098-1004(1996)8:1<38::AIDHUMU5>3.0.CO;2-L.

47. Froissart R, Guffon N, Vanier MT, Desnick RJ, Maire I. Fabry disease: D313Y is an alpha-galactosidase a sequence variant that causes pseudodeficient activity in plasma. Mol Genet Metab. 2003. https://doi.org/10.1016/S10967192(03)00136-7.

48. Ishii S, Nakao S, Minamikawa-Tachino R, Desnick RJ, Fan J-Q. Alternative splicing in the alpha-galactosidase a gene: increased exon inclusion results in the Fabry cardiac phenotype. Am J Hum Genet. 2002. https://doi.org/10. 1086/339431.

49. Adzhubei I, Jordan DM, Sunyaev SR. Predicting functional effect of human missense mutations using PolyPhen-2. Curr Protoc Hum Genet. 2013. https://doi.org/10.1002/0471142905.hg0720s76.

\section{Publisher's Note}

Springer Nature remains neutral with regard to jurisdictional claims in published maps and institutional affiliations.

Ready to submit your research? Choose BMC and benefit from:

- fast, convenient online submission

- thorough peer review by experienced researchers in your field

- rapid publication on acceptance

- support for research data, including large and complex data types

- gold Open Access which fosters wider collaboration and increased citations

- maximum visibility for your research: over $100 \mathrm{M}$ website views per year

At BMC, research is always in progress.

Learn more biomedcentral.com/submissions 